\title{
The Role of TWIST in Angiogenesis and Cell Migration in Giant Cell Tumor of Bone
}

\author{
Shalini Singh, Isabella W. Y. Mak, Divya Handa, and Michelle Ghert \\ Department of Surgery, McMaster University, 711 Concession Street, B3 Surgical Offices, Hamilton, ON, Canada L8V 1C3
}

Correspondence should be addressed to Michelle Ghert; michelle.ghert@jcc.hhsc.ca

Received 13 February 2014; Revised 7 April 2014; Accepted 22 April 2014; Published 14 May 2014

Academic Editor: Julie Teruya-Feldstein

Copyright (C) 2014 Shalini Singh et al. This is an open access article distributed under the Creative Commons Attribution License, which permits unrestricted use, distribution, and reproduction in any medium, provided the original work is properly cited.

Giant cell tumor of bone (GCT) is a bone tumor consisting of numerous multinucleated osteoclastic giant cells involved in bone resorption and neoplastic osteoblast-like stromal cells responsible for tumor growth. The tumor occasionally metastasizes to the lung; however, factors leading to metastasis in this tumor are unknown. The TWIST-1 protein (also referred to as TWIST) has been suggested to be involved in epithelial-mesenchymal transition (EMT) and tumor progression in some cancers. In this study we investigated the functional role of TWIST in GCT cell angiogenesis and migration. Overexpression of TWIST in neoplastic GCT stromal cells significantly increased mRNA and protein expression of VEGF and VEGFR1 in vitro, whereas knockdown of TWIST resulted in decreased VEGF and VEGFR1 expression. A stable cell line with TWIST overexpression resulted in features of EMT including increased cell migration and downregulation of E-cadherin. The results of our study indicate that TWIST may play an important role in angiogenesis and cell migration in GCT.

\section{Introduction}

Giant cell tumor of bone (GCT) is an aggressive bone tumor characterized by the presence of an abundance of reactive multinucleated giant cells surrounded by mesenchymal stromal cells. To date, the oncogenesis of GCT remains unknown as the neoplastic stromal cells appear to be preosteoblastic cells that do not undergo terminal osteoblastic differentiation [1-4]. GCT is a highly vascular tumor and in several cases metastasizes to the lungs $[2,5,6]$.

Bone tumors recruit new blood vessels from preexisting vessels of the host through factors secreted from either the tumor itself or the surrounding stromal cells $[7,8]$. Tumor growth is dependent upon the growth of these new blood vessels. Angiogenesis is the process of developing new capillaries from the existing circulation via a complex multistep system regulated by a delicate balance between various angiogenic inhibitors and activators [9]. Activators of angiogenesis include growth factors, such as vascular endothelial growth factor (VEGF), platelet-derived growth factor (PDGF), and hypoxic conditions that activate hypoxia-inducible factor-1 (HIF-1) which in turn upregulate angiogenic proteins as well as angiogenic oncogenes [9].
The transcription factor TWIST is known to induce an embryonic event termed epithelial-mesenchymal transition (EMT) in tumor cells [10]. EMT is a process initially observed in embryonic development in which the cells lose epithelial cell properties and gain mesenchymal cells characteristics [11]. In hepatocellular carcinoma, TWIST overexpression has been shown to induce angiogenesis, inhibit apoptosis, and increase tumor aggressiveness [12].

Previous work in our lab has shown that TWIST is overexpressed at the transcription level in GCT patient specimens [13]. In the present study we expanded these results and reported that TWIST upregulates angiogenic factors and subsequent cell migration in GCT. Thus, TWIST acts as an upstream regulator of the angiogenic and migration associated aggressiveness of this bone tumor.

\section{Materials and Methods}

2.1. Primary Cell Lines Culture. We established primary cell cultures of GCT stromal tumor cells from fresh GCT tissue obtained from three patients following ethics board approval and patient consent. The tissue was processed and maintained in DMEM containing 10\% FBS, 2 mM glutamine, and 
$100 \mathrm{U} / \mathrm{mL}$ antibiotics. The resulting cell suspension together with macerated tissue was cultured at $37^{\circ} \mathrm{C}$ in humidified air with $5 \% \mathrm{CO}_{2}$. Following several successive passages, the mesenchymal stromal cells became the homogeneous cell type, whereas the multinucleated giant cells were eliminated from culture.

2.2. Plasmids Construction and Transfection. A TWIST open reading frame was amplified by PCR from a cDNA TWIST clone (Origene, Rockville, MD) using oligonucleotides cgcggatccgcgatgatgcaggacgtgtcc and ccggaattccggctagtgggacgcgacat, containing BamHI and EcoRI restriction sites, respectively. After BamHI and EcoRI digestion, the open reading frame was ligated into a pcDNA3.1 vector into TWIST plasmids.

GCT cells were transfected using an electroporation method $[13,14]$. Briefly, cells were trypsinized and washed with PBS and resuspended into $1 \mathrm{~mL}$ FBS free media with $35 \mu \mathrm{g}$ of TWIST plasmid. Cells with TWIST plasmid mixture were electroporated using the Gene Pulser II electroporation apparatus (Bio-Rad) with optimized combinations of voltage and capacitance. The cells were then plated in $10 \mathrm{~cm}$ Petri dishes with supplemented DMEM. After $48 \mathrm{~h}$ posttransfection, cells were harvested for RNA isolation.

2.3. siRNA Transfection. Mesenchymal stromal cells of GCT were trypsinized and transfected with TWIST small interfering RNAs (siRNAs) via electroporation. Stromal cells of GCT were washed and resuspended in Optimem reduced serum media (Gibco, Invitrogen, Canada). Subsequently, the cell suspension was mixed with either $200 \mathrm{nM}$ of TWIST siRNA (Invitrogen), a positive Silencer GAPDH siRNA control, or a nonspecific negative control (Ambion Inc.). Stromal cells with the siRNA mixture were electroporated using the Gene Pulser II electroporation apparatus (Bio-Rad Laboratories) under a single-pulse protocol with optimized combination of voltage and capacitance. Cells were then plated in $10 \mathrm{~cm}$ cell culture dishes with supplemental DMEM. After $48 \mathrm{~h}$ of transfection, cells were harvested for RNA isolation. RPS18 was selected among other housekeeping genes for normalization in real time PCR analysis and GAPDH was used as a positive siRNA control.

2.4. RNA Isolation and cDNA Synthesis. Total RNA extraction was performed according to the manufacturer's protocol (QIAGEN Total RNA Kit). Concentration and purity of the samples were evaluated by the ratio of optical density 260:280 using a spectrophotometer (Beckman DU 530). One microgram of total RNA was used for cDNA synthesis following the manufacturer's instructions (qScript cDNA Supermix, Quanta BioSciences) under this setting: $25^{\circ} \mathrm{C}$ for $5 \mathrm{~min}, 42^{\circ} \mathrm{C}$ for $30 \mathrm{~min}$, and then $95^{\circ} \mathrm{C}$ at $5 \mathrm{~min}$.

2.5. Quantitative RT-PCR. The cDNA samples were measured using the quantitative real time PCR (qRT-PCR) (MJ Mini Gradient Thermocycler, Bio-Rad). Cycle threshold (Ct) numbers were derived from the exponential phase of PCR amplification. Relative changes in mRNA expression were calculated using the comparative $\Delta \Delta \mathrm{CT}$ (crossing point) method. For comparison of transcript levels between samples, a standard curve of cycle thresholds for serial dilutions of a cDNA sample was established and then used to calculate the relative abundance of transcripts of each gene. Values were then normalized to the relative amounts of RPS18 cDNA. All PCRs were performed in triplicate experiments. Primer sequences are listed in Table 1.

2.6. Migration Assays. Migration assays were performed as described earlier [15]. Briefly, an equal number $(1 \times$ $10^{5}$ ) of GCT empty vector-transfected cells and TWIST transfected cells (GCTT) were resuspended in serum-free medium and plated in the top compartment of a standard $8 \mu \mathrm{m}$ pore $\mathrm{BD}$ falcon cell culture insert. Bottom wells were filled with $5 \%$ serum medium. Following a $24 \mathrm{~h}$ period of invasion at $37^{\circ} \mathrm{C}$, invaded cells to the bottom wells were detached with cell detachment buffer, lysed, and stained with crystal violet dye. Dyed cells were counted using a light microscope.

2.7. Wound Closure Assay. Cells were grown in $35 \mathrm{~mm}$ culture plates (Falcon) to 70-80\% confluent level in DMEM medium containing 10\% fetal bovine serum. Multiple uniform streaks $(\sim 50 \mu \mathrm{m}$ width) were made on the monolayer culture with $100 \mu \mathrm{L}$ pipette tips. Streaked plates were immediately washed with DMEM medium with $10 \%$ FBS to remove detached cells. Mitomycin ( $5 \mu \mathrm{g} / \mathrm{mL}$; Sigma) was used in the medium to inhibit proliferation of GCT cells for determination of the cell migration. Cell migration was monitored for $24 \mathrm{~h}$, and pictures were taken at $0 \mathrm{~h}$ and $24 \mathrm{~h}$ time points with a digital camera attached to an inverted microscope. Six to eight fields were analyzed, and the mean percentage of wound distance covered by cells was calculated. The distance of wound width was expressed in microns before and after migration.

2.8. Western Blotting. Cytoplasmic fractions were isolated from these transfected cells by scraping after $24 \mathrm{~h}$ of incubation and then centrifuged for $5 \mathrm{~min}$. The cells were lysed with NP-40 containing lysis buffer (10 mM Tris, pH 7.4, $10 \mathrm{mM}$ $\mathrm{NaCl}, 5 \mathrm{mM} \mathrm{MgCl}_{2}$, and $0.5 \% \mathrm{NP}-40$ ) to disrupt the cell membrane and then the cell lysate was centrifuged at $500 \mathrm{~g}$ for $5 \mathrm{~min}$ at $4^{\circ} \mathrm{C}$. The supernatant (cytoplasmic fraction) was removed and the pellet (nuclear fraction) was resuspended in NP-40 containing cell lysis buffer. Proteins were denatured by boiling in sample buffer, separated on 12\% SDS-PAGE, transferred onto the PVDF membrane (Immobilon TM-PSQ, Millipore), and blocked overnight in 5\% nonfat powdered milk in TBST (10 mM Tris-HCl, pH 7.5, $100 \mathrm{mM} \mathrm{NaCl}$, and $0.1 \%(\mathrm{v} / \mathrm{v})$ Tween-20). The membrane was incubated in primary mouse anti-MMP2 antibody (1:1000, Calbiochem), mouse anti-TWIST, (1:1000, Abcam), E-cadherin (1:700, Santa Cruz), and VEGF (1:1000, Millipore) overnight at $4^{\circ} \mathrm{C}$. Following membrane washes in $1 \mathrm{X}$ TBS-T, the membrane was incubated in the HRP-conjugated secondary anti-mouse antibody (1:10,000, Promega) for 1 hour at RT followed by protein visualization on audioradiography film. 
TABLE 1: Human primer sequences designed for real time PCR amplification.

\begin{tabular}{lcc}
\hline Gene & Primers & Primer sequence \\
\hline VEGF & F & ATCTTCAAGCCATCCTGTGTG \\
& R & CAAGGCCCACAGGGATTTTC \\
RPS18 & F & GATGGGCGGCGGAAAATA \\
& R & GCGTGGATTCTGCATAATGGT \\
E-Cadherin & F & CGGGAATGCAGTTGAGGATC \\
& R & AGGATGGTGTAAGCGATGGC \\
Fibronectin & F & CGGTGGCTGTCAGTCAAAG \\
& R & AAACCTCGGCTTCCTCCATAA \\
MMP-2 & F & ACATCAAGGGCATTCAGGAG \\
& R & ACATCAAGGGCATTCAGGAG \\
VEGFR1 & F & CACCAAGAGCGACGTGTG \\
& R & TTTTGGGTCTCTGTGCCAG \\
TWIST & F & TACATCGACTTCCTCTACCAGGTC \\
& R & TAGTGGGACGCGGACATGGA \\
\hline
\end{tabular}

2.9. Statistical Analysis. Statistical analyses for the real time PCR were performed using the two-sample independent Student's $t$-test. The average value within each experiment was expressed relative to the expression of internal control gene; $P$ value of $<0.05$ was considered to be statistically significant.

\section{Results}

3.1. Overexpression of TWIST Activates the Expression of Angiogenic Factors. In order to determine whether TWIST plays a role in angiogenesis through VEGF expression in GCT stromal cells, we generated 3 stable TWIST overexpressing cell lines (GCTT1 and GCTT2 and GCTT3) from three different patient specimens using a TWIST-pcDNA3.1 construct and compared them to pcDNA3.1 empty vector. Quantitative real time PCR showed that overexpression of TWIST significantly increased the mRNA expression of VEGF and VEGFR1 in GCT stromal cells (Figure 1(a), $P<$ $0.05)$. These results were confirmed by western blot analysis showing an increase in protein expression (Figure 1(b)).

3.2. Suppression of Angiogenic Factors in Response to TWIST Knockdown. TWIST siRNA was used to knockdown TWIST expression in GCT stromal cells. Quantitative RT-PCR revealed that knockdown of TWIST resulted in a significant decrease in VEGF and VEGFR1 expression in GCT stromal cells (Figure 2(a), $P<0.01$ ). GAPDH siRNA was used as a positive control to determine transfection efficiency. GAPDH mRNA was reduced to approximately $70 \%$ of the original expression level when treated with GAPDH siRNA but was found unaffected when treated with random and TWIST siRNA (data not shown). Western blot analysis confirmed decreased protein expression of VEGF with TWIST knockdown (Figure 2(b)).
3.3. TWIST Induces EMT in GCT Stromal Cells. We next explored the relationship between TWIST and epithelialmesenchymal transition (EMT) in GCT stromal cells. EMT is an important mechanism associated with cancer invasiveness and metastasis $[10,16]$. It is a process characterized by loss of cell adhesion, repression of E-cadherin expression, and increased cell motility. To determine the role of TWIST in invasiveness and metastasis of GCT, we generated TWIST overexpressing GCT cell lines using a TWIST-pcDNA3.1 construct. We observed that overexpression of TWIST significantly decreased the expression of E-cadherin (an epithelial marker) and increased the expression of fibronectin (an extracellular matrix protein) and MMP-2 (EMT mediator) at the mRNA expression and protein levels in GCT stromal cells (Figures 3(a) and 3(b)). As expected, knockdown of TWIST in the GCT stromal cells induced E-cadherin expression as shown in both quantitative real time PCR and western blot analysis (Figures 3(c) and 3(d)). We further examined the subcellular localization of E-cadherin in the TWIST-depleted GCT stromal cells compared to the untransfected GCT cells. We observed that the E-cadherin expression in untransfected cells (GCT-UT) was faint and diffused throughout the cell; however, TWIST knockdown resulted in more localized and dense staining of E-cadherin on the surface of the cell where it would be expected to be functionally active (Figure 3(e)).

3.4. TWIST Promotes Migration in GCT Stromal Cells. To examine whether overexpression of TWIST promotes migratory activity in GCT stromal cells, cell wound-healing and migration assays were performed using untransfected (UT) and TWIST transfected (GCTT) GCT stromal cell lines. The wound-healing assay showed significantly faster cell movement in GCTT than in the UT control group (Figure 4(a)). Overexpression of TWIST increased the migratory ability of GCT cells by nearly twofold when compared to untransfected cells $(P<0.01)$ (Figure $4(\mathrm{~b}))$. The pattern was consistent across both GCT cell lines studied.

We further studied the migratory potential of cells using Boyden's chamber assay. Cells were seeded into the transwell insert and migrated through pores of the membrane at the bottom of insert with 5\% FBS gradient over the period of $24 \mathrm{~h}$ incubation. Migrated stained cells were counted and represented graphically (Figure 4(c)). We observed an increase of over $200 \%$ in migration in the TWIST transfected (GCTT) cells when compared to the control GCT transfected with the empty vector (GCT-EV) (Figure 4(d)).

\section{Discussion}

GCT is a highly vascular and locally invasive tumor with metastatic potential [17-19]. In this study we investigated an important but previously unreported signaling pathway that regulates angiogenesis and EMT in GCT. We observed that TWIST regulates the expression of angiogenic factors by the tumor cells and also regulates EMT features such as Ecadherin expression and cell migration. 


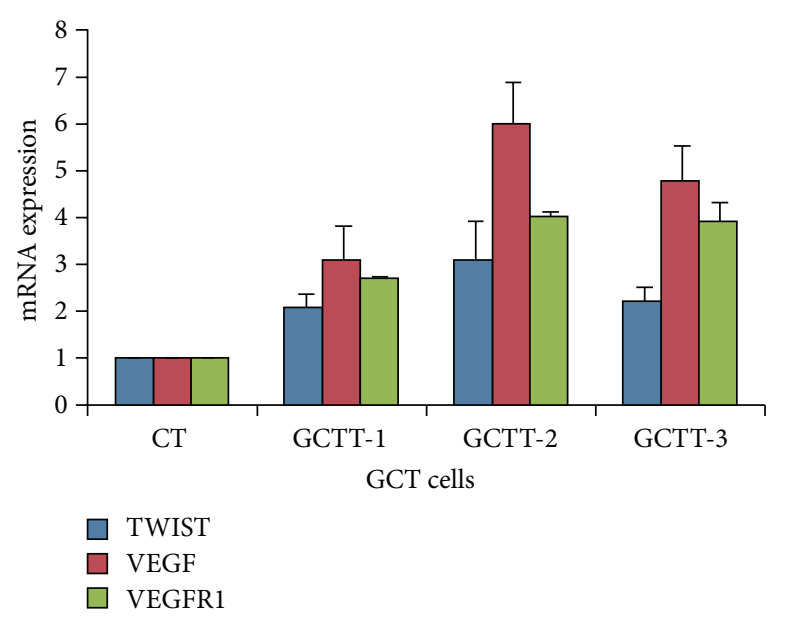

(a)

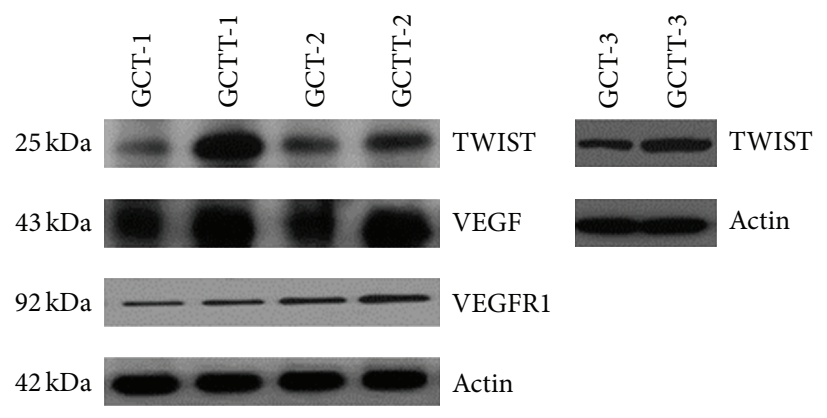

(b)

FIGURE 1: TWIST overexpression in GCT cells. (a) qRT-PCR analysis shows an increase in TWIST, VEGF, and VEGFR1 expression $(P<0.05)$ in all 3 GCT cells transfected with a TWIST pcDNA3.1 construct compared to empty vector transfected GCT control. (b) These results were verified at the protein level by western blot analysis.

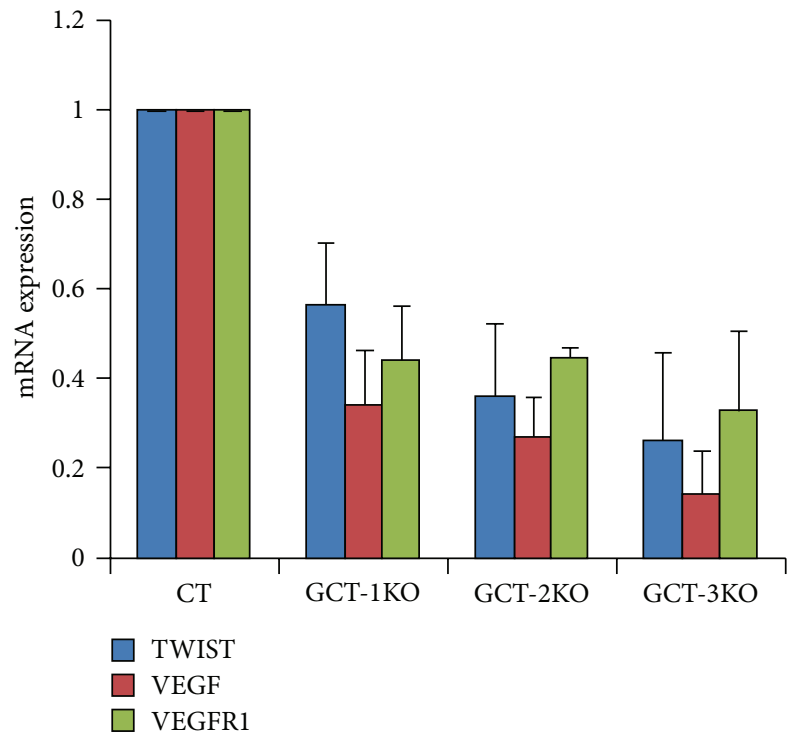

(a)

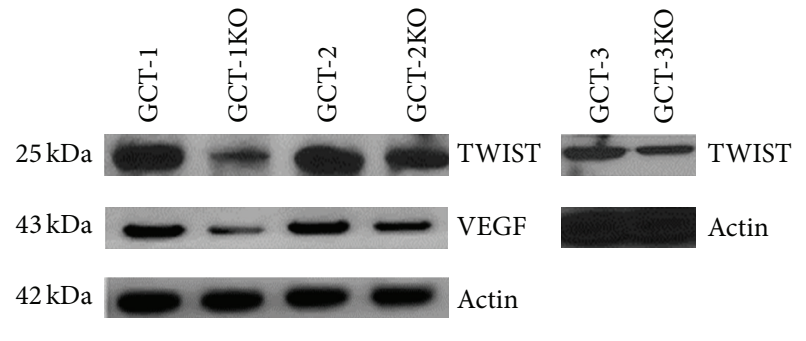

(b)

FIGURE 2: The effect of TWIST siRNA on VEGF expression in the mesenchymal stromal cells of GCT. (a) The expression of TWIST in untreated (UT) and TWIST knockdown (GCTKO) GCT cells was determined using qRT-PCR in triplicate. TWIST knockdown resulted in a statistically significant decrease in VEGF and VEGFR1 expression in GCT cells $(P<0.01)$. (b) Western blot also shows a decrease in VEGF, VEGFR1, and TWIST protein expression in GCT stromal cells transfected with TWIST siRNA compared to untreated GCT cells.

Previous work in our lab reported that TWIST, a master osteogenic regulator, was highly expressed in all GCT specimens examined [13, 14]. TWIST is a basic helix-loop-helix (bHLH) protein that plays a central role not only in EMT but also in cell type determination and differentiation and has been shown to regulate early osteogenesis [20]. TWIST has been shown to regulate EMT indirectly through binding to the E-cadherin promoter and suppressing transcription $[16,21,22]$. Here we found that, in GCT cells, TWIST indeed regulates E-cadherin expression and increases the migration of the tumor cells. Thus, although TWIST may have a role in the osteoblastic differentiation of the tumor cells in GCT, it is also likely to play a role in the aggressive characteristics of the tumor cells.

TWIST overexpression in an MCF-7 xenograft in vivo breast cancer model has been shown to increase tumor growth and angiogenesis [23]. We considered the possibility that overexpression of TWIST results in downstream angiogenesis and blood vessel recruitment in GCT. TWIST expression in both nuclei and cytoplasm of GCT osteoblastic 


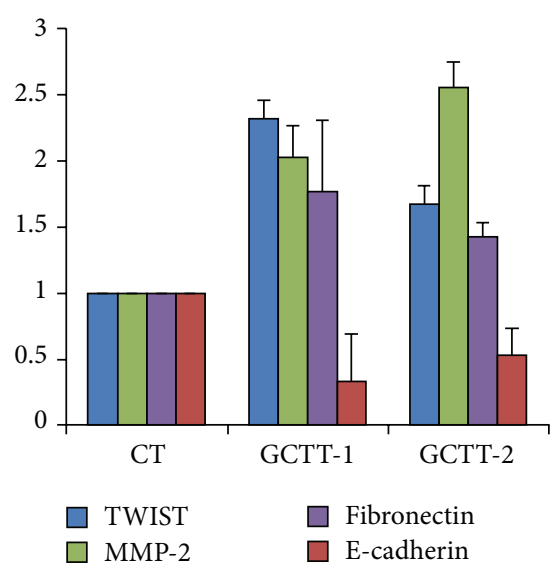

(a)

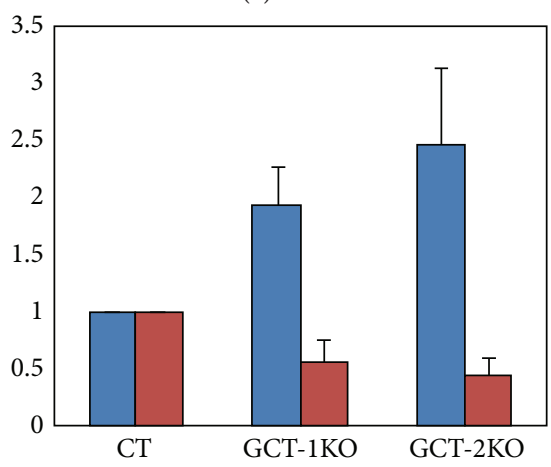

E-cadherin

TWIST-1 (c)

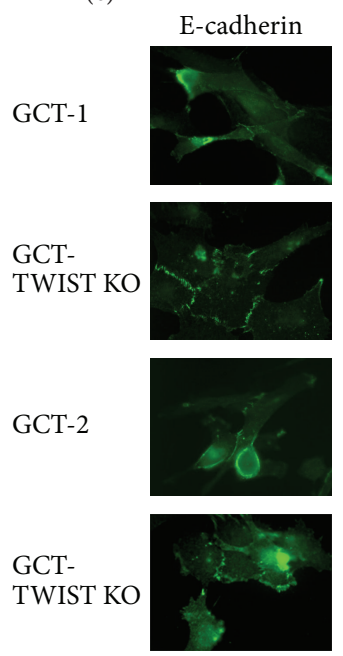

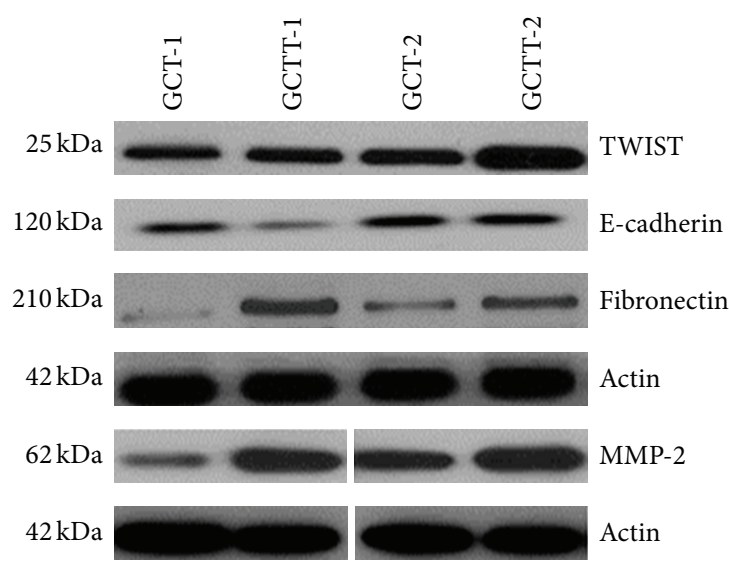

(b)

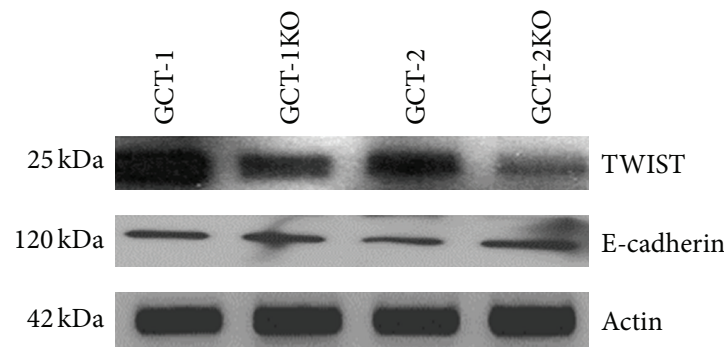

(d)
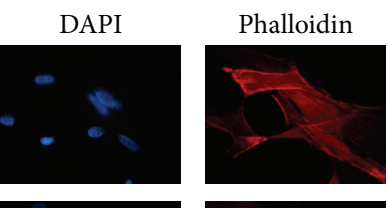

Composite
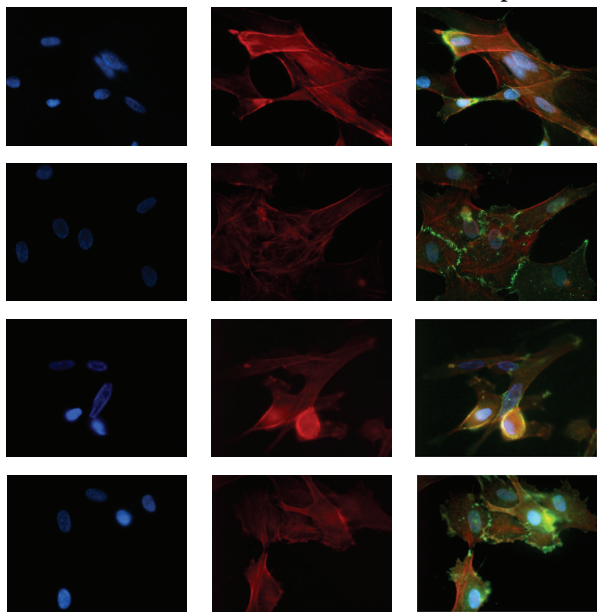

(e)

FIgURE 3: The effect of TWIST siRNA on E-cadherin expression in the mesenchymal stromal cells of GCT. (a) The expression of TWIST in untreated (CT) and TWIST overexpressed (GCTT) GCT cells was determined using qRT-PCR in triplicate. TWIST overexpression resulted in a statistically significant decrease in E-cadherin expression and increase in fibronectin and MMP-2 expression at the transcript level $(P<$ 0.01). (b) Validation of transcript data by western blots. (c) The expression of empty vector treated (UT) and TWIST knockdown (GCT KO) GCT cells was determined using qRT-PCR in triplicate. TWIST knockdown resulted in a statistically significant increase in E-cadherin expression in GCT cells $(P<0.01)$. (d) Western blot demonstrated the same trend in TWIST and E-cadherin protein levels in GCT stromal cells transfected with TWIST siRNA compared to untransfected GCT cells. (e) Subcellular localization of E-cadherin in TWIST-depleted and GCT untransfected stromal cells was detected by immunofluorescence using an anti-E-cadherin antibody. DAPI staining (blue) indicates the nuclei, and phalloidin (red) is used for cytoskeleton staining (oil immersion; 100x magnification). 


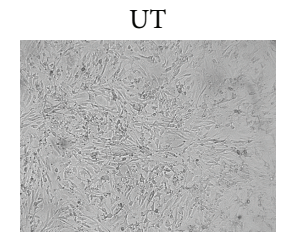

GCTT
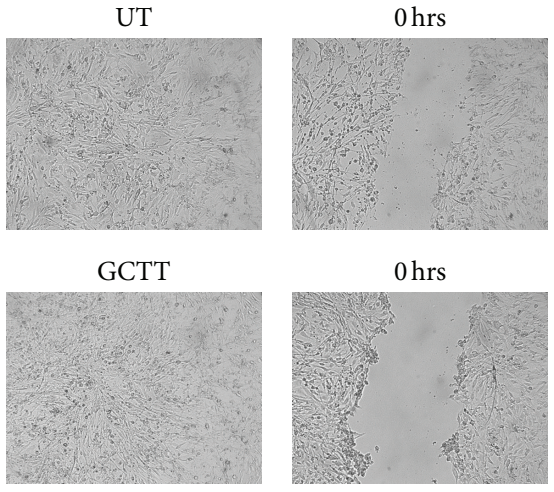

0 hrs

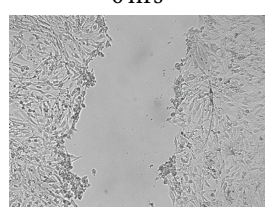

(a)

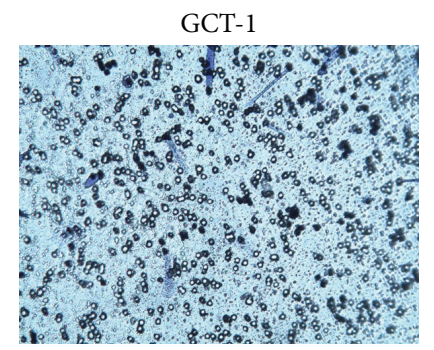

GCT-2
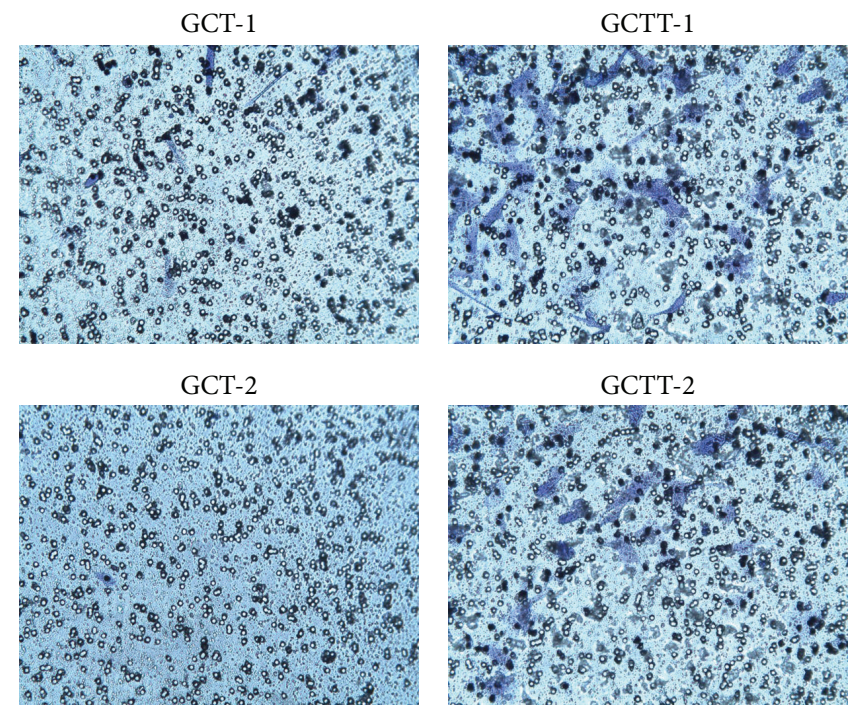

GCTT-2

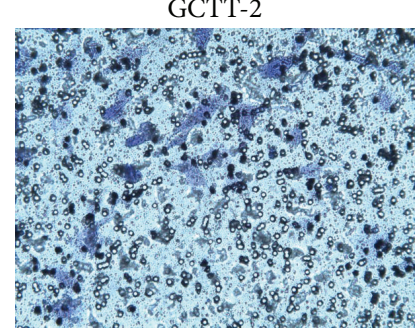

(c)

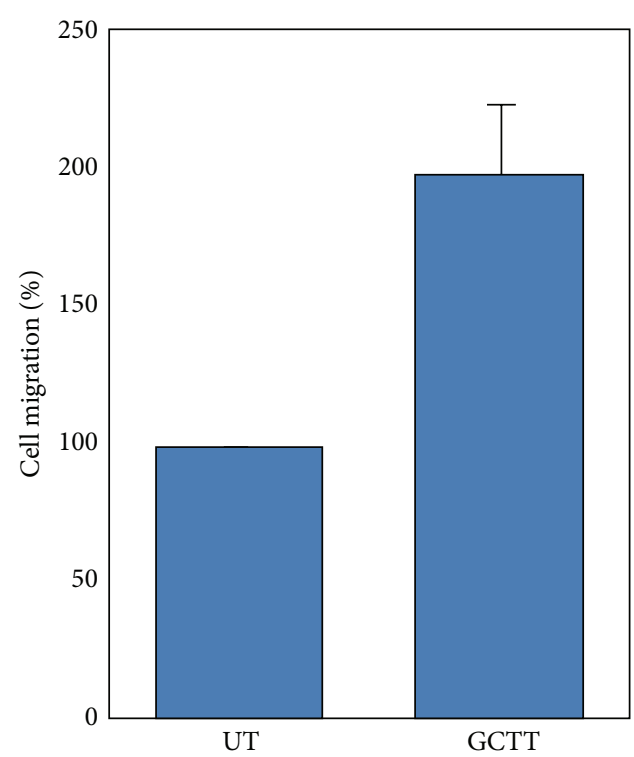

(b)

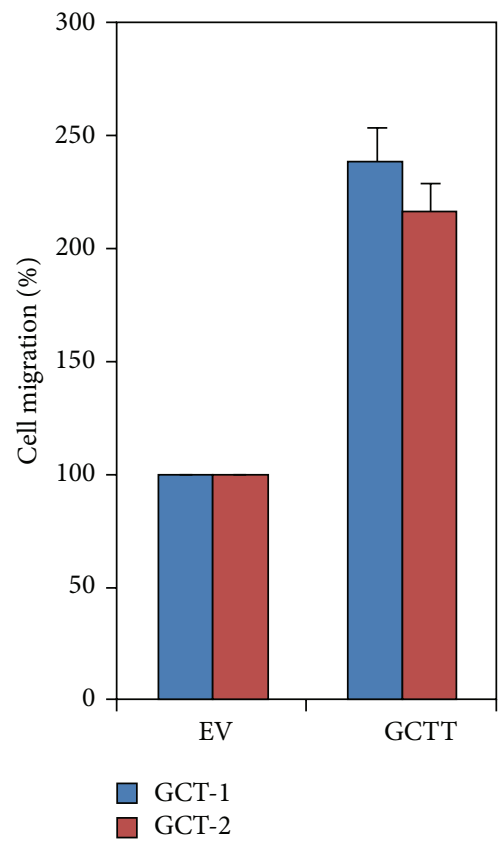

(d)

FIGURE 4: TWIST promotes migration in GCT cells. (a) Wound-healing assay: GCT untransfected (UT) and TWIST transfected (GCTT) cells were scraped and measured after $24 \mathrm{~h}$. The "wounded" distances were examined at the indicated time points under 100x magnification. (b) Cell migration was calculated in percentage compared to the control UT. (c) Cell migration assay: migration assays were performed using GCT cells transfected with a TWIST overexpression vector (GCTT) and the empty vector (EV) control cells in transwell plates. Shown are representative pictures of the cell migration assay after staining the cells from the underside of the insert. (d) Cell migration based on cell density on the undersurface of the insert is shown graphically.

cells indicates the possible role of TWIST in angiogenesis and tumor progression. Several studies have indicated that tumor cells secrete MMPs with an extracellular matrix that contributes to angiogenesis and malignancy in early and advanced stages of tumors $[24,25]$. It has been reported that higher expression of VEGF is associated with the aggressiveness of the GCT tumor [17]. We also observed an increase in VEGF and VEGFR1 expression with overexpression of TWIST, whereas knockdown of TWIST expression resulted in decreased expression of these angiogenic factors. These findings indicate that TWIST plays an upstream role in angiogenesis in GCT.

Others have reported that the mechanism by which TWIST promotes tumor invasion and metastasis is likely 
to be via the inhibition of E-cadherin and increased cell migration $[10,26]$. We observed that overexpression of TWIST in GCT transfected cells indeed resulted in EMT changes such as suppression of E-cadherin expression and increased fibronectin expression. Fibronectin plays a key role in the tissue remodeling and cell migration events that occur during normal embryonic development and adult wound-healing. Fibroblasts secrete proteases, including matrix metalloproteinases (MMPs). MMPs act as critical mediators of the EMT process, which stimulates cell proliferation, cell migration, and cell morphological alternation $[17,24,25]$. We further observed that ectopic TWIST overexpression activates the MMP-2 expression at transcript and protein levels which indicates that MMPs could stimulate intrinsic cancer cells and stroma cell responses to tumor malignancy.

Moreover, knockdown of TWIST expression inhibited cell migration in an in vitro assay and accordingly induced E-cadherin expression. Moreover, knockdown of TWIST expression inhibited cell migration and also induced Ecadherin expression. We further performed wound-healing and transwell insert chamber assays to examine the effect of TWIST on metastasis potential. Our results clearly demonstrate that TWIST overexpression in GCT cells induced cell migration in vitro. Suppression of TWIST expression in highly metastatic mammary carcinoma cells specifically inhibits its metastatic ability [10, 27]; therefore, the role that TWIST plays in GCT cell migration and angiogenesis may indicate its involvement in tumor metastasis.

In summary, we have demonstrated that TWIST promotes tumor angiogenic factor expression and suppression of E-cadherin expression. TWIST, therefore, regulates processes promoting tumor growth such as EMT, migration, angiogenesis, and possibly metastasis. Such coordinated regulation of tumor growth and metastasis warrants consideration for the development of therapeutic targeted intervention.

\section{Conclusion}

In this study, an important but previously unreported signaling pathway that regulates angiogenesis and EMT in GCT was investigated. We observed that, in GCT stromal cells, TWIST regulates the expression of the angiogenic factor VEGF and the EMT biomarker E-cadherin, as well as cell migration. TWIST has been shown to regulate EMT indirectly through binding to the E-cadherin promoter and suppressing transcription in breast cancer cells. Therefore the high expression of TWIST in GCT may result in downstream angiogenesis and blood vessel recruitment as well as cell migration, both markers of tumor aggressiveness.

TWIST promotes tumor angiogenic factor expression and suppression of E-cadherin expression in GCT stromal cells. TWIST, therefore, regulates processes promoting tumor growth such as EMT, migration, angiogenesis, and possibly metastasis. Such coordinated regulation of tumor growth and metastasis warrants consideration for the development of therapeutic targeted intervention.

\section{Conflict of Interests}

The authors declare that there is no conflict of interests regarding the publication of this paper.

\section{Acknowledgments}

The authors would like to thank these following funding sources: Juravinski Cancer Centre Foundation Grant and McMaster University Surgical Associates Grant. The funders had no role in study design, data collection and analysis, decision to publish, or preparation of the paper.

\section{References}

[1] M. Asagiri and H. Takayanagi, "The molecular understanding of osteoclast differentiation," Bone, vol. 40, no. 2, pp. 251-264, 2007.

[2] M. Ghert, N. Simunovic, R. W. Cowan, N. Colterjohn, and G. Singh, "Properties of the stromal cell in giant cell tumor of bone," Clinical Orthopaedics and Related Research, no. 459, pp. 8-13, 2007.

[3] S. R. Goldring, A. L. Schiller, and H. J. Mankin, "Characterization of cells from human giant cell tumors of bone," Clinical Orthopaedics and Related Research, vol. 204, pp. 59-75, 1986.

[4] M. Nishimura, K. Yuasa, K. Mori et al., "Cytological properties of stromal cells derived from giant cell tumor of bone (GCTSC) which can induce osteoclast formation of human blood monocytes without cell to cell contact," Journal of Orthopaedic Research, vol. 23, no. 5, pp. 979-987, 2005.

[5] E. Katz, M. Nyska, and E. Okon, "Growth rate analysis of lung metastases from histologically benign giant cell tumor of bone," Cancer, vol. 59, no. 10, pp. 1831-1836, 1987.

[6] D. J. McDonald, F. H. Sim, R. A. McLeod, and D. C. Dahlin, "Giant-cell tumor of bone," Journal of Bone and Joint Surgery. American, vol. 68, no. 2, pp. 235-242, 1986.

[7] J. Folkman, "Tumor angiogenesis: a possible control point in tumor growth," Annals of Internal Medicine, vol. 82, no. 1, pp. 96-100, 1975.

[8] J. Folkman, "What is the evidence that tumors are angiogenesis dependent?" Journal of the National Cancer Institute, vol. 82, no. 1, pp. 4-6, 1990.

[9] P. Nyberg, L. Xie, and R. Kalluri, "Endogenous inhibitors of angiogenesis," Cancer Research, vol. 65, no. 10, pp. 3967-3979, 2005.

[10] J. Yang, S. A. Mani, J. L. Donaher et al., “Twist, a master regulator of morphogenesis, plays an essential role in tumor metastasis," Cell, vol. 117, no. 7, pp. 927-939, 2004.

[11] B. Thisse, M. E. Messal, and F. Perrin-Schmitt, "The twist gene: isolation of a Drosophila zygotle gene necessary for the establishment of dorsoventral pattern," Nucleic Acids Research, vol. 15, no. 8, pp. 3439-3453, 1987.

[12] R. F. Niu, L. Zhang, G. M. Xi et al., "Up-regulation of twist induces angiogenesis and correlates with metastasis in hepatocellular carcinoma," Journal of Experimental and Clinical Cancer Research, vol. 26, no. 3, pp. 385-394, 2007.

[13] S. Singh, I. W. Y. Mak, R. W. Cowan, R. Turcotte, G. Singh, and M. Ghert, "The role of TWIST as a regulator in giant cell tumor of bone," Journal of Cellular Biochemistry, vol. 112, no. 9, pp. 2287-2295, 2011. 
[14] S. Singh, M. Singh, I. W. Mak, R. Turcotte, and M. Ghert, "Investigation of FGFR2-IIIC signaling via FGF-2 ligand for advancing GCT stromal cell differentiation," PLoS ONE, vol. 7, Article ID e46769, 2012.

[15] I. W. Mak, R. E. Turcotte, and M. Ghert, "Parathyroid hormonerelated protein (PTHrP) modulates adhesion, migration and invasion in bone tumor cells," Bone, vol. 55, no. 1, pp. 198-207, 2013.

[16] J. Fu, L. Qin, T. He et al., “The TWIST/Mi2/NuRD protein complex and its essential role in cancer metastasis," Cell Research, vol. 21, no. 2, pp. 275-289, 2011.

[17] S. M. Kumta, L. Huang, Y. Y. Cheng, L. T. C. Chow, K. M. Lee, and M. H. Zheng, "Expression of VEGF and MMP-9 in giant cell tumor of bone and other osteolytic lesions," Life Sciences, vol. 73, no. 11, pp. 1427-1436, 2003.

[18] Y. Matsumoto, Y. Okada, J.-I. Fukushi et al., "Role of the VEGFFlt-1-FAK pathway in the pathogenesis of osteoclastic bone destruction of giant cell tumors of bone," Journal of Orthopaedic Surgery and Research, vol. 5, no. 1, article 85, 2010.

[19] R. M. Taylor, T. G. Kashima, H. J. Knowles, and N. A. Athanasou, "VEGF, FLT3 ligand, PlGF and HGF can substitute for M-CSF to induce human osteoclast formation: implications for giant cell tumour pathobiology," Laboratory Investigation, vol. 92, pp. 1398-1406, 2012.

[20] M. S. Lee, G. N. Lowe, D. D. Strong, J. E. Wergedal, and C. A. Glackin, "TWIST, a basic helix-loop-helix transcription factor, can regulate the human osteogenic lineage," Journal of Cellular Biochemistry, vol. 75, pp. 566-577, 1999.

[21] F. Vesuna, P. van Diest, J. H. Chen, and V. Raman, “Twist is a transcriptional repressor of E-cadherin gene expression in breast cancer," Biochemical and Biophysical Research Communications, vol. 367, no. 2, pp. 235-241, 2008.

[22] M.-H. Yang, D. S.-S. Hsu, H.-W. Wang et al., "Bmil is essential in Twistl-induced epithelial-mesenchymal transition," Nature Cell Biology, vol. 12, no. 10, pp. 982-992, 2010.

[23] Y. Mironchik, P. T. Winnard Jr., F. Vesuna et al., “Twist overexpression induces in vivo angiogenesis and correlates with chromosomal instability in breast cancer," Cancer Research, vol. 65, no. 23, pp. 10801-10809, 2005.

[24] A. John and G. Tuszynski, "The role of matrix metalloproteinases in tumor angiogenesis and tumor metastasis," Pathology and Oncology Research, vol. 7, no. 1, pp. 14-23, 2001.

[25] S.-O. Yoon, S.-J. Park, C.-H. Yun, and A.-S. Chung, "Roles of matrix metalloproteinases in tumor metastasis and angiogenesis," Journal of Biochemistry and Molecular Biology, vol. 36, no. 1, pp. 128-137, 2003.

[26] L. Zhang, M. Yang, L. Gan et al., "DLX4 upregulates TWIST and enhances tumor migration, invasion and metastasis," International Journal of Biological Sciences, vol. 8, pp. 1178-1187, 2012.

[27] T. K. Lee, R. T. P. Poon, A. P. Yuen et al., "Twist overexpression correlates with hepatocellular carcinoma metastasis through induction of epithelial-mesenchymal transition," Clinical Cancer Research, vol. 12, no. 18, pp. 5369-5376, 2006. 

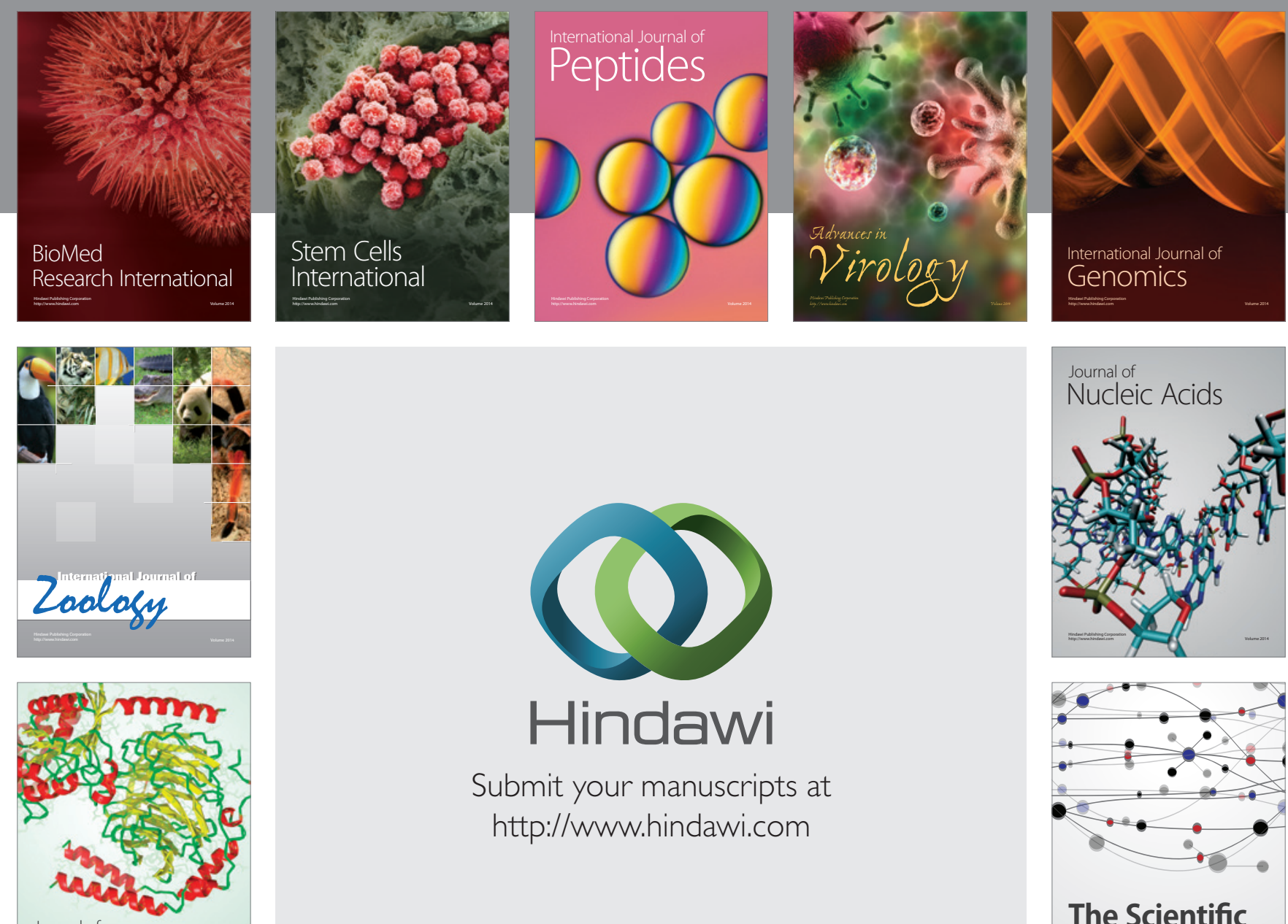

Submit your manuscripts at

http://www.hindawi.com

Journal of
Signal Transduction
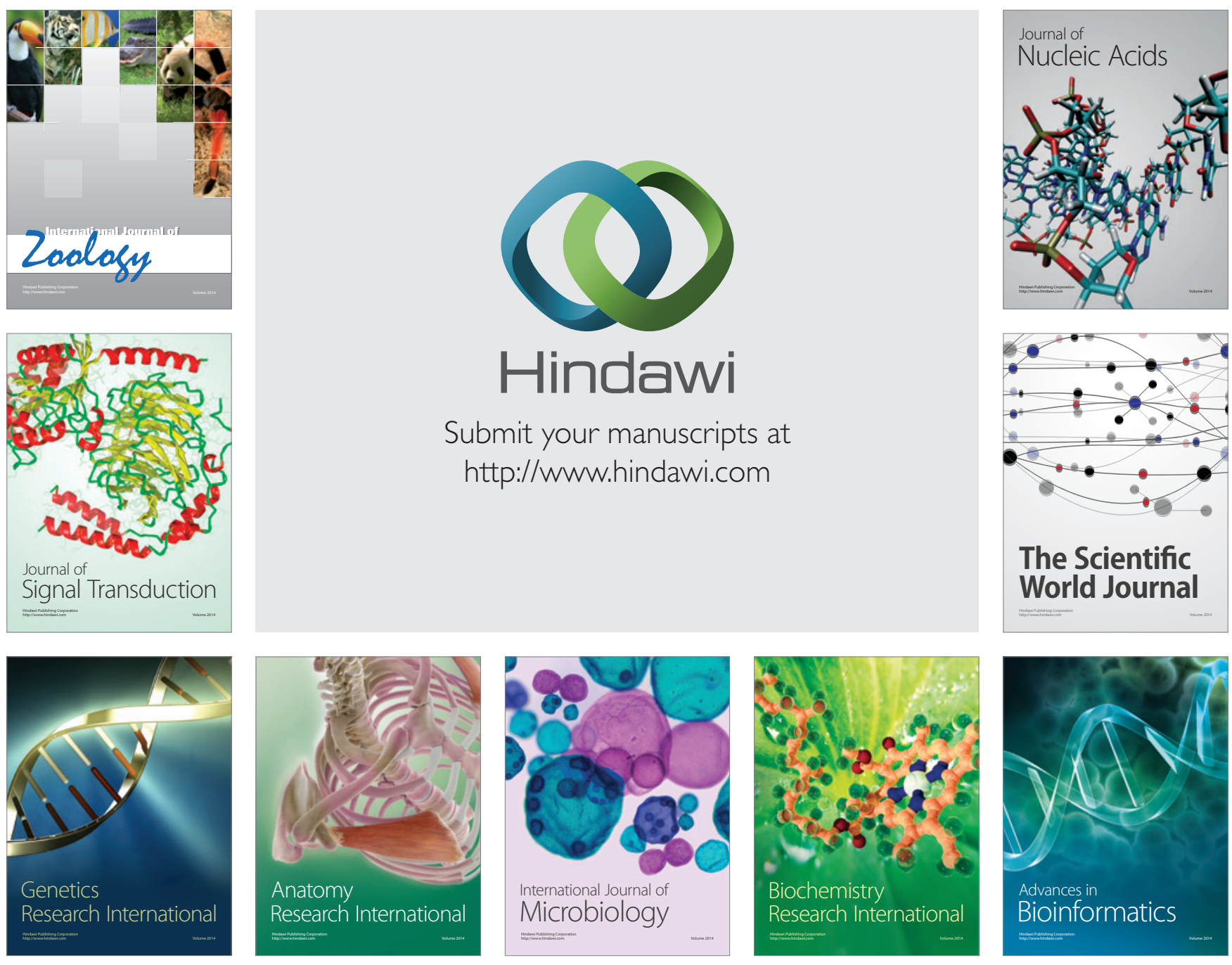

The Scientific World Journal
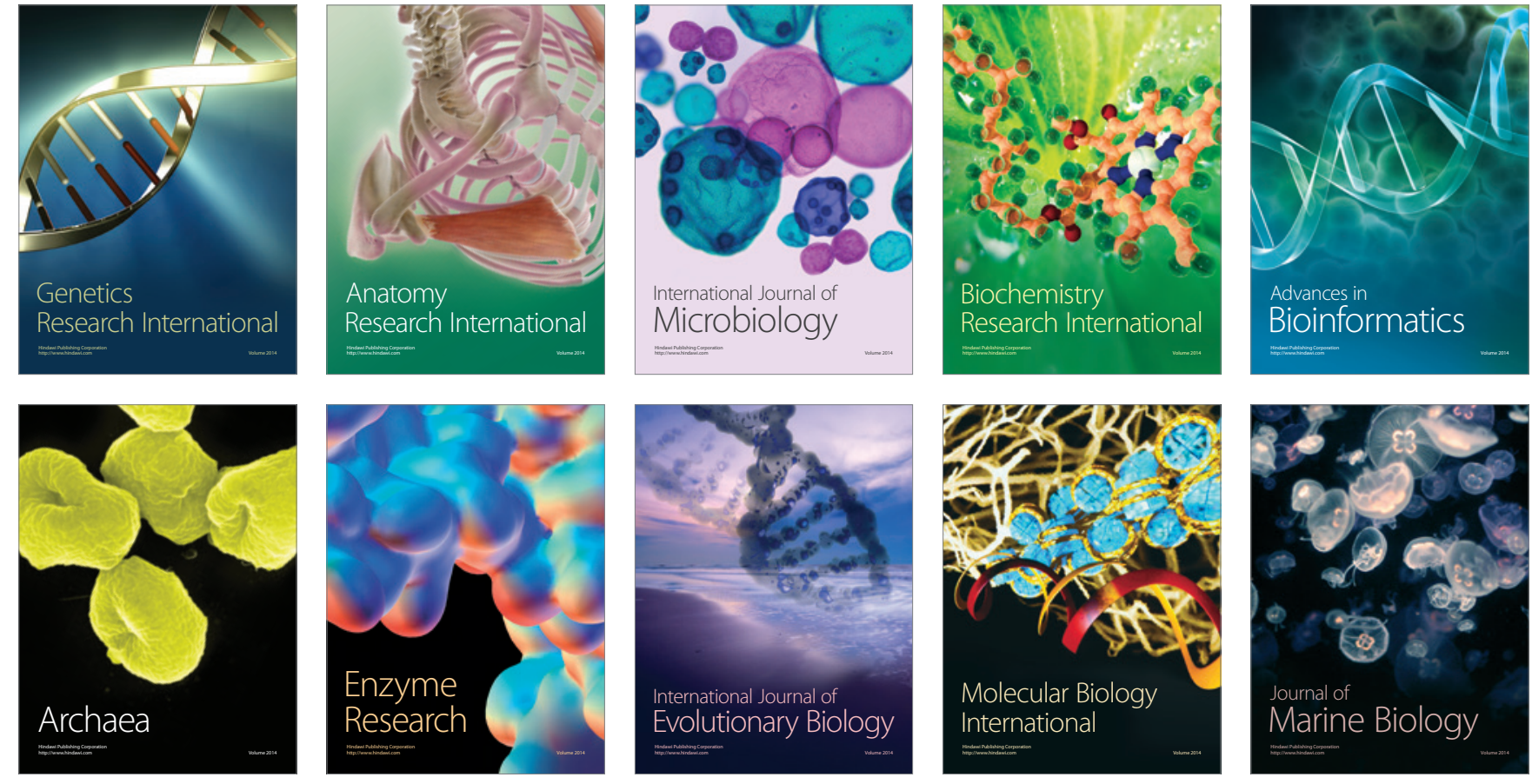